\title{
Detection of construction errors in ex vivo coronary artery anastomoses by 13-MHz epicardial ultrasonography
}

Ricardo P. J. Budde, MSc, Rudy Meijer, Thomas C. Dessing, MSc, Cornelius Borst, MD, PhD, and

Paul F. Gründeman, MD, PhD

From Heart Lung Center Utrecht, University Medical Center, Utrecht, The Netherlands.

Received for publication July 4, 2004; revisions received Sept 1, 2004; accepted for publication Sept 7, 2004

Address for reprints: Paul F. Gründeman, $\mathrm{MD}, \mathrm{PhD}$, Associate Professor, Cardiothoracic and Vascular Research, Large Animals, University Medical Center Utrecht (Room G02.523), Heidelberglaan 100, 3584 CX Utrecht, The Netherlands (Email: utrecht.cardioresearch@hli.azu.nl and p.f.grundeman@hli.azu.nl).

J Thorac Cardiovasc Surg 2005;129: $1078-83$

$0022-5223 / \$ 30.00$

Copyright () 2005 by The American Association for Thoracic Surgery

doi:10.1016/j.jtcvs.2004.09.002
Objective: Intraoperative detection of suboptimal coronary anastomoses allows revision before chest closure. We evaluated an epicardial $13-\mathrm{MHz}$ ultrasound minitransducer as a means to detect three different coronary anastomosis construction errors.

Methods: In total, 120 internal thoracic artery-to-coronary artery anastomoses were constructed correctly $(n=60)$ or incorrectly $(n=60)$ with one technical error: suture crossover, purse-string or deep toe stitch $(\mathrm{n}=20$ each). Anastomoses were performed on ex vivo pressure-perfused porcine (96 anastomoses) and human hearts (24 anastomoses). Two blinded observers scanned and scored the anastomoses with epicardial ultrasonography. In 24 human and 24 porcine anastomoses, angiograms were made of 24 correct and 24 incorrect anastomoses and scored by two other blinded observers. Angioscopy and cast injection served as a reference.

Results: Overall, 119 of 120 anastomoses were accurately scored as correct or incorrect within a median of 67 seconds (8-381 seconds) by both observers (sensitivity 0.98 , specificity $1.00, \kappa 1.00(1.00,1.00$, and 1.00 in angiography subset, respectively). One deep toe stitch that induced outflow corner stenosis was spotted by both observers but regarded as insignificant and thus inaccurately scored as correct. In 5 anastomoses, unintended irregularities were detected. By angiography, anastomoses were accurately scored with a sensitivity of 0.75 and a specificity of $0.81(P<.001$ vs ultrasonography) and $\kappa$ of 0.54 . Angioscopy and cast confirmed ultrasonographic findings and did not reveal irregularities other than detected by ultrasonography.

Conclusion: Ex vivo epicardial 13-MHz ultrasonography allowed rapid and accurate evaluation of coronary anastomoses and detected technical construction errors with higher sensitivity and specificity than angiography.

$\mathrm{T}$ here is concern about the increased risk of anastomosis construction errors in (minimally invasive) off-pump coronary artery bypass grafting (CABG) because the suturing process is technically more demanding than surgery on the arrested heart. ${ }^{1}$ In on-pump CABG, total occlusion or stenosis greater than $50 \%$ is seen in as many as $9 \%$ of grafts at short-term angiographic follow-up. ${ }^{1}$ Angioscopy reveals irregularities and technical errors that include purse-string effect, intimal flaps, thrombus formation, and misplaced sutures in as many as $24 \%$ of anastomoses. ${ }^{2,3}$

An intraoperative technique to assess anastomotic quality could improve patency rates in all approaches to $\mathrm{CABG}$, because suboptimal anastomoses might be revised before chest closure. ${ }^{4}$ Currently, angiography is the gold standard, but it is rarely used intraoperatively. ${ }^{5}$ Epicardial ultrasonography for anastomosis visualization and assessment was described in the 1980 s. $^{6}$ Despite promising results, ${ }^{6}$ technical limitations prevented widespread use. Recently, we described a new high-frequency epicardial ultrasound minitransducer $(15 \times 9 \times 6 \mathrm{~mm})$ for assessment of the left 
anterior descending coronary artery (LAD) in patients ${ }^{7}$ and geometry assessment of anastomoses. ${ }^{8,9}$ Because of its small size and high frequency (up to $13 \mathrm{MHz}$ in B-mode), this minitransducer has potential for routine intraoperative assessment of anastomoses at all locations on the heart.

The aim of this study was to investigate the ability of two blinded observers to detect and characterize three different standardized construction errors in coronary anastomoses on ex vivo hearts with the $13 \mathrm{MHz}$ minitransducer and compare the results against the gold standard of angiography.

\section{Material and Methods}

\section{Ex Vivo Porcine and Postmortem Human Hearts}

On 46 ex vivo porcine hearts, 96 internal thoracic artery (ITA)to-LAD anastomoses were constructed. On 4 isolated postmortem human hearts, a total of 24 anastomoses were constructed on the $\mathrm{LAD}$, diagonal branches, right coronary artery, and circumflex coronary artery. All grafts were porcine ITAs. The coronary arteries were cannulated proximally to pressurize $(80 \mathrm{~mm} \mathrm{Hg})$ the anastomoses with saline solution through a Langendorff setup.

\section{Anastomosis Construction}

All 120 anastomoses were randomly constructed by a single investigator (R.P.J.B.) deliberately either correctly $(n=60)$ or with a single, standardized construction error $(n=60)$, either suture crossover, purse-string, or deep toe stitch $(\mathrm{n}=20$ each). The distribution of anastomosis types is summarized in Table 1. The crossover anastomosis was constructed by interlocking two suture bites on opposite sides of the arteriotomy approximately a third of the anastomotic orifice length from the toe. The purse-string anastomosis was made by pulling heavily on the suture ends before fashioning the suture. In the deep toe suture bite anastomosis, the suture was passed through the posterior wall of the coronary artery at the toe.

Anastomotic sites on the porcine LAD were chosen at random. In the human hearts (24 anastomoses), the anastomosis was deliberately placed in sites with $(\mathrm{n}=12)$ and without $(\mathrm{n}=12)$ atherosclerotic disease, as determined by digital palpation and epicardial ultrasonographic scanning (Table 1).

\section{Ultrasonographic Equipment}

As in our previous work, ${ }^{8,9}$ a commercially available, highfrequency (up to $13 \mathrm{MHz}$ in B-mode), linear-array minitransducer $(15 \times 6 \times 9 \mathrm{~mm}$; Aloka Co, Ltd, Tokyo, Japan $)$ was used. The image scan width was $10 \mathrm{~mm}$. The minitransducer was placed in a handling tool that could be held like a pencil. ${ }^{7}$ Imaging was performed with an Aloka SSD 5000 Prosound ultrasound system (Aloka Co, Ltd). Ultrasound transmission gel (Parker Laboratories, Fairfield, NJ) was applied directly onto the anastomosis for proper contact.

\section{Ultrasonographic Scanning}

Two observers (R.M. and T.C.D.) blinded to the anastomosis type scanned and scored all anastomoses as described. Both had extensive experience in echocardiographic scanning of coronary anas-
TABLE 1. Number and distribution of anastomosis types

\begin{tabular}{llcc}
\hline Anastomosis type & $\begin{array}{l}\text { Porcine } \\
\text { hearts }\end{array}$ & $\begin{array}{c}\text { Human } \\
\text { hearts }\end{array}$ & Total \\
\hline Control & $48(12)$ & $12(12)\{6\}$ & $60(24)\{6\}$ \\
Suture crossover & $16(4)$ & $4(4)\{2\}$ & $20(8)\{2\}$ \\
Purse-string & $16(4)$ & $4(4)\{2\}$ & $20(8)\{2\}$ \\
Deep toe stitch & $16(4)$ & $4(4)\{2\}$ & $20(8)\{2\}$ \\
Total & $96(24)$ & $24(24)\{12\}$ & $120(48)\{12\}$
\end{tabular}

Numbers in parentheses represent anastomosis subsets in which angiography was performed; numbers in braces represent anastomosis subset constructed in vessel area with plaque.

tomoses. Scan time needed to obtain sufficient information for scoring was recorded.

\section{Angiography}

In a subgroup of 24 porcine anastomoses (12 correct, 4 of each type of error) and all 24 human heart anastomoses (12 correct, 4 of each type of error), angiography (C-arm, Pulsera; Philips Nederland BV Medical Systems, Eindhoven, The Netherlands) of the graft and anastomosis was performed in at least two different oblique projections by selectively injecting contrast media through the ITA (Table 1). Two independent observers (J.M.P.G.E. and C.B.) blinded to the anastomosis type scored the angiograms as described.

\section{Anastomosis Scoring by Ultrasonography and Angiography}

Anastomoses were scored as either correct or a construction error. The location and appearance of detected technical errors and irregularities were indicated in a schematic drawing of the anastomosis. The location was categorized in one or more of the following categories: ITA, anastomotic orifice, inflow corner, outflow corner, and recipient coronary artery.

\section{Angioscopy and Cast}

During perfusion, a 2.4-mm angioscope (11281A; Karl Storz $\mathrm{GmbH} \& \mathrm{Co}$, Tüttlingen, Germany) was introduced through the free proximal end of the ITA to visualize the anastomosis. ${ }^{2,3}$ A polyvinylsiloxane impression material (Kerr Co, Romulus, Mich) was injected into the ITA to fill the coronary artery, the anastomosis, and the ITA itself. The resulting 3-dimensional cast of the anastomosis was removed from the vessels after hardening and visually inspected at $3.5 \times$ magnification. Angioscopic and cast findings combined served as a reference for ultrasonographic and angiographic findings.

\section{Statistical Analysis}

The $\kappa$ value was calculated to rate the agreement between observers and interpreted according to Landis and Koch. ${ }^{10}$ Sensitivity and specificity were calculated for the scoring of both observers combined. Scores were compared with the $\chi^{2}$ test. Scan times are presented as median with range and were compared with the Wilcoxon rank sum test. 


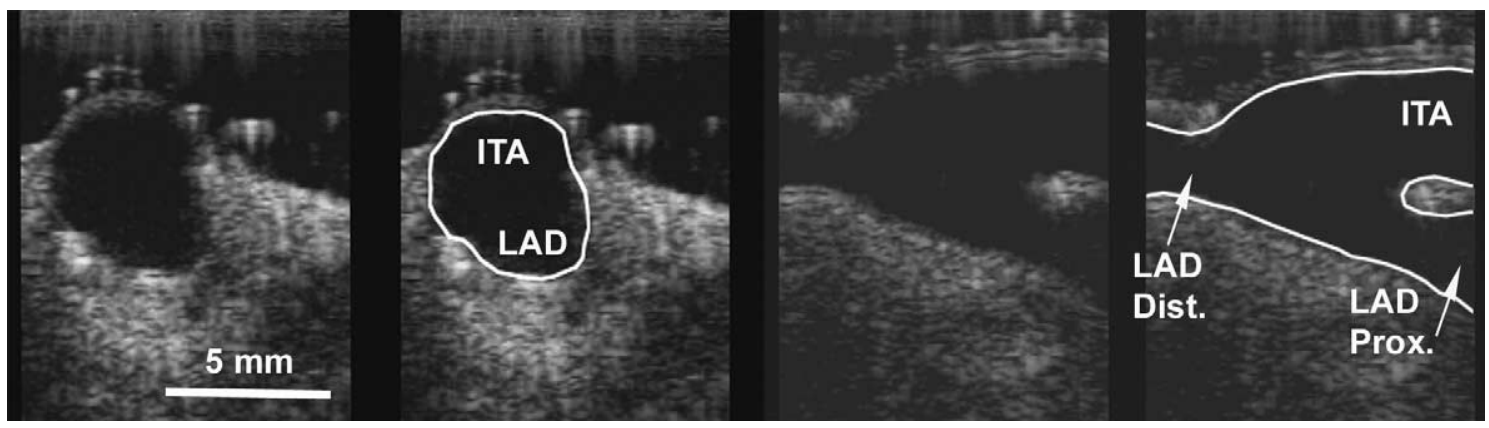

Figure 1. Correctly constructed control anastomosis: transverse (left panels) and longitudinal ultrasonographic (right panels) images. Dist, Distal; Prox, proximal.

\section{Results}

\section{Anastomosis Construction}

External inspection could not discriminate incorrect from correct anastomoses.

\section{Ultrasonographic Scanning and Scoring}

All 120 anastomoses were easily visualized within a median of 67 seconds (range 8-381 seconds). Visualization of the porcine anastomoses (median 61 seconds, range 8-381 seconds) was faster than that of the human anastomoses (median 92 seconds, range 14-256 seconds, $P<.001$ ). Representative ultrasonographic images of a control anastomosis are presented in Figure 1.

Both observers accurately scored 119 of 120 (99\%; Table 2) anastomoses as correct or construction error (sensitivity 0.98 , specificity $1.00, \kappa 1.00$ ). In most anastomoses,

\section{TABLE 2. Scoring results of both observers combined}

\begin{tabular}{llll}
\hline Scoring & Ultrasonography & Angiography & $P$ value \\
\hline
\end{tabular}

Judgment of construction error versus correct overall

$\begin{array}{ll}\text { Accuracy } & 99 \% \\ \text { Sensitivity } & 0.98 \\ \text { Specificity } & 1.00 \\ \kappa & 1.00\end{array}$

Judgment of construction error versus correct in angiography group

$\begin{array}{lccc}\text { Accuracy } & 100 \% & 78 \% & <.001 \\ \text { Sensitivity } & 1.00 & 0.75 & <.001 \\ \text { Specificity } & 1.00 & 0.81 & <.001 \\ \kappa & 1.00 & 0.54 & -\end{array}$

Location of error overall Accuracy $\quad 100 \%$

Location of error in angiography group

$\begin{array}{llll}\text { Accuracy } & 100 \% & 68 \% & -\end{array}$

Percentages are means of the percentages of the two observers. the presence of a construction error was detected directly on first visualization of the anastomosis. In the single inaccurately scored anastomosis (deep toe stitch anastomosis), slight narrowing of the outflow corner was spotted but considered insignificant and thus scored as correct by both observers.

Each of the construction errors had a distinct appearance on the ultrasonographic image. The crossover anastomosis had an echodense spot in the anastomotic orifice (Figure 2). In the purse-string anastomosis, the anastomotic orifice was narrowed (Figure 3). In the deep toe stitch anastomosis, narrowing of the outflow corner was seen (Figure 4). All errors were seen in both the longitudinal and transverse scan planes.

One purse-string anastomosis appeared the same as a control anastomosis on ultrasonography and was scored as such by both observers. In 5 anastomoses, unintended irregularities were detected by both observers: outflow corner narrowing with LAD disruption in a purse-string anastomosis, additional purse-string effect of various degrees in 3 anastomoses, and an inflow corner narrowing in 1 anastomosis. All these findings were confirmed by angioscopy and cast and analyzed as such.

In several anastomoses, tissue was detected in the anastomosis only by the second observer. It is conceivable that sometimes tissue was introduced during the angioscopy procedure that was performed between scans by the two observers. This is a known complication of angioscopy. ${ }^{2}$ Those findings were not used in the analysis.

In all anastomoses scored as construction error, the error location was accurately scored by both observers $(\kappa 1.0)$. The sort of error was correctly identified in 58 of 60 anastomoses by observer 1 and in 54 of 60 anastomoses by observer 2 (93\% overall).

Presence of calcifications in the human hearts was not a confounding factor for image interpretation. Calcifications showed clear echocardiographic shadowing (Figure 5), making them easy to discriminate from construction errors. 


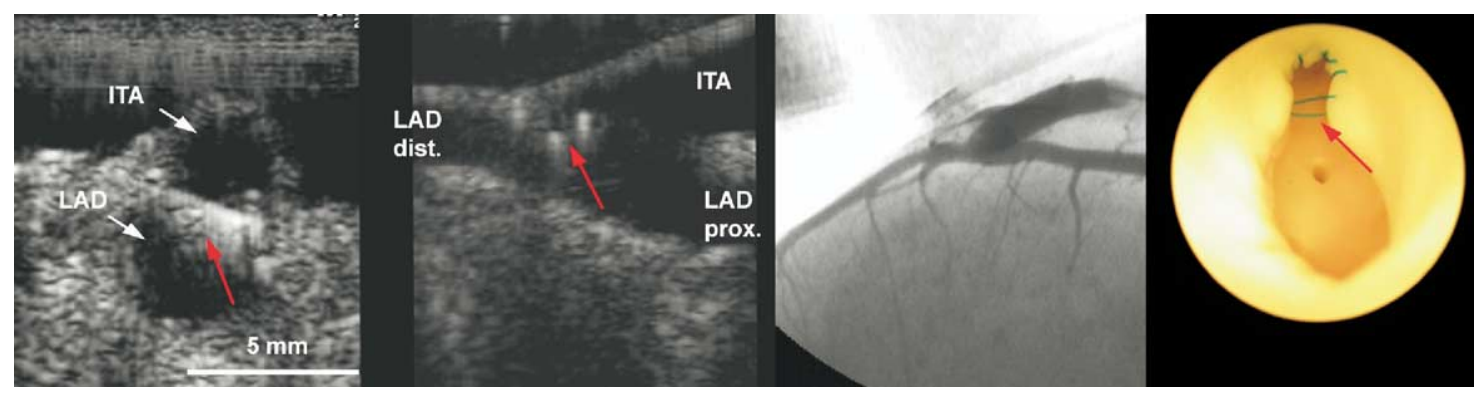

Figure 2. Suture crossover anastomosis: panels from left to right, transverse and longitudinal ultrasonographic images, angiogram, and angioscopic image taken from ITA toward outflow corner. Note in the longitudinal ultrasonographic image the suture traversing the anastomotic orifice twice (arrow). The transverse image was taken at the level of the overcrossing suture. The anastomosis appeared normal on the angiogram. Dist, Distal; Prox, proximal.

\section{Angiography}

Of the 48 anastomoses in which angiography was performed, 36 and 39 were accurately scored as correct or construction error by observers 3 and 4, respectively (sensitivity 0.75 , specificity $0.81, \kappa 0.54$; Table 2 ). In these 48 anastomoses, the sensitivity and specificity of ultrasonography were both 1.0 ( $P<.001$ vs angiography). The suture crossover error proved to be particularly difficult to detect by angiography (Figure 2). Of the inaccurately scored anastomoses, 5 were inaccurately scored by both observers.

The error location in anastomoses accurately scored as construction error was accurately identified in 10 of 18 and 14 of 18 anastomoses (68\% accurate overall) by observers 3 and 4 , respectively. Three anastomoses were inaccurately scored because of the presence or presumed presence of plaque and calcification.

Angiographically, the specific kind of construction error could not be determined reliably. Only a distinction between outflow corner and orifice narrowing could be made.

\section{Angioscopy and Cast}

Angioscopy and cast findings corresponded with ultrasonographic findings, and no irregularities other than those detected by ultrasonography were noted.

\section{Discussion}

The principal findings of this study were as follows: (1) Epicardial ultrasonography enabled detection of construction errors in coronary anastomoses with significantly higher sensitivity and specificity than angiography. (2) With epicardial ultrasonography, the location and type of error could be accurately determined in $100 \%$ (vs $68 \%$ by angiography) and $93 \%$, respectively, of the anastomoses in which an error was detected. (3) Ultrasonographic scanning required only a median of 67 seconds for assessment of an anastomosis.

\section{Anastomosis Quality Control}

With the advent of off-pump and minimally invasive CABG, there has been a renewed interest in techniques for intraoperative assessment of distal anastomosis quality. ${ }^{4,11,12}$ Currently, only graft flow measurement is used on a large scale. ${ }^{11}$ Graft flow measurement, however, can only detect severe stenosis $(>75 \%)$ and provides no information about its location. Furthermore, there is no clear cutoff point for adequate graft flow, and thus it may underestimate the number of suboptimal anastomoses. For various reasons, the gold standard of angiography is not used frequently, most importantly because it is invasive, not readily available in the operating room, and time-consuming. ${ }^{5}$
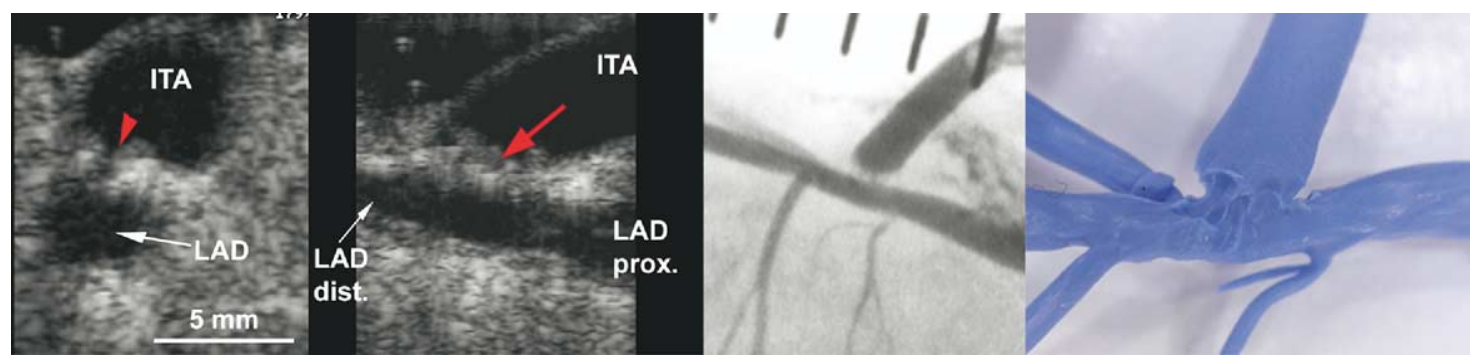

Figure 3. Purse-string anastomosis: panels from left to right, transverse and longitudinal ultrasonographic images, angiogram, and cast. Note in the transverse and longitudinal ultrasonographic images severe narrowing of the anastomotic orifice (arrow) and the small resulting lumen (arrowhead). Dist, Distal; Prox, proximal. 


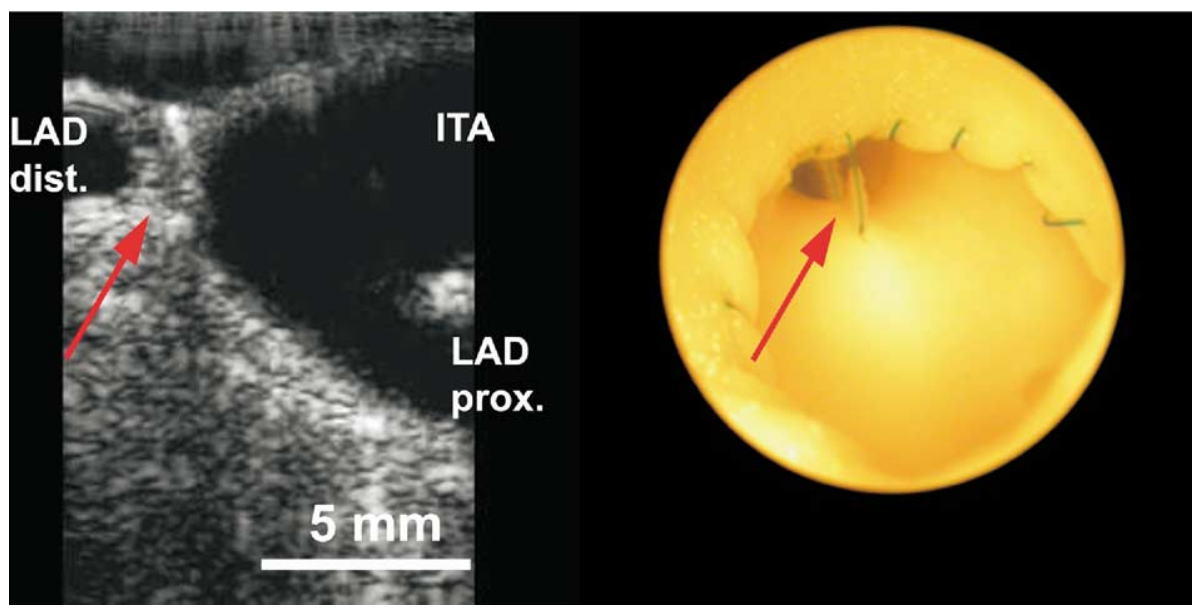

Figure 4. Deep toe stitch anastomosis: longitudinal ultrasonographic image (left panel) and angioscopic image from ITA toward outflow corner (right panel). Note in the angioscopic image the suture passing through the posterior wall of the LAD (arrow). Dist, Distal; Prox, proximal.

The potential of epicardial ultrasonography for coronary anastomosis assessment was recognized almost 20 years ago. ${ }^{6}$ Technical transducer limitations, however, have prevented widespread clinical introduction. The current minitransducers allow easier access. To date, no detailed evaluation has been reported regarding the ability of epicardial ultrasonography to detect and characterize different technical errors in coronary anastomoses relative to the gold standard of angiography.

In the subgroup of 48 anastomoses evaluated with angiography, epicardial ultrasonography enabled detection of con- struction errors with significantly higher sensitivity (1.00) and specificity (1.00) than achieved with angiography (0.75 and 0.81 , respectively). When calculated from all 120 anastomoses in the study, the sensitivity and specificity of epicardial ultrasonography were 0.98 and 1.00 , respectively.

In addition, epicardial ultrasonography enabled discrimination between narrowing caused by suture errors or by calcifications, that present with a distinct echo shadowing (Figure 5). This was not possible with angiography.

Anastomosis evaluation with epicardial ultrasonography required only a median of 92 seconds in human anastomo-

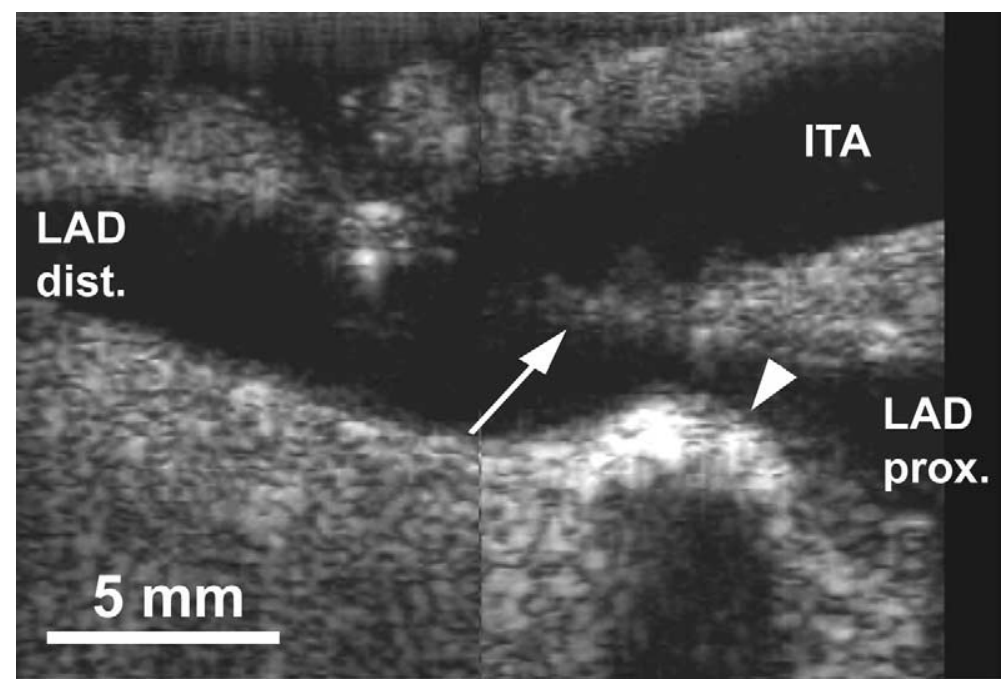

Figure 5. Composite longitudinal ultrasonographic image of calcification in a purse-string anastomosis (arrowhead). Note its bright appearance and distinct echo shadowing. In the anastomotic orifice, the lateral wall can be seen because of narrowing by the purse-string effect (arrow). Dist, Distal; Prox, proximal. 
ses, which is clinically acceptable. In most cases, the detection of the error was instantaneous, and subsequent scanning time was spent characterizing the error and looking for additional irregularities. Clinically, revision would be performed directly after a serious error was spotted.

As we have shown before, ${ }^{7}$ the minitransducer can also be used to locate the coronary artery and select the optimal anastomotic site. Combined with its use for anastomotic quality assessment and possibly epiaortic scanning as well, the ultrasound minitransducer may prove a multipurpose diagnostic tool to improve the quality of CABG surgery.

A concern with the use of epicardial ultrasonography for anastomosis quality assessment is that image interpretation is subjective and operator dependent. ${ }^{4}$ In this study, however, the two observers scored all anastomoses identically with regard to the presence of a construction error $(\kappa 1.0)$, indicating an almost perfect agreement, ${ }^{10}$ whereas the $\kappa$ for angiography was 0.54 , indicating only moderate agreement. The observers did have extensive previous experience with the interpretation of ultrasonographic images of anastomoses. Preferably, the surgeon would be trained in a laboratory setting, including scanning of anastomoses on ex vivo hearts perfused with saline solution and image interpretation of off-line images on a computer. This might take a couple of hours. Alternatively, the surgeon might teach himself or herself by starting to acquire images during cardioplegic arrest and later studying the images off-line. This would permit peer review by a radiologist.

\section{Limitations}

The experimental setup in ex vivo hearts presents several limitations relative to the in vivo situation. First, exposure of the anastomosis for ultrasonographic scanning was not hampered by motion artifacts. We have experience, however, with scanning ITA-LAD anastomoses on the beating porcine heart. Image quality has been comparable to that in this study and has allowed successful detection of anastomotic irregularities in comparable scan times. ${ }^{9}$ Second, buildup of angiographic contrast in the ex vivo hearts as a result of lack of washout made the angiograms less easy to interpret. Third, 96 of 120 anastomoses were constructed on healthy porcine coronary vessels. However, 12 of the 24 human anastomoses were deliberately constructed in a diseased part of the coronary artery. The plaque and calcification did not impair the ability to assess the anastomosis by ultrasonography.

\section{Conclusion}

With an epicardial 13-MHz ultrasound minitransducer, three technical errors in coronary anastomoses constructed ex vivo were detected in about 1 minute with a higher sensitivity (1.00) and specificity (1.00) than with angiography ( 0.75 and 0.81 , respectively). Epicardial ultrasonography is a promising technique for routine, clinical, intraoperative, noninvasive quality control of coronary anastomoses.

We acknowledge the technical contributions of C. W. J. Verlaan and M. Emons and the statistical advice of M. Schipper, PhD. We thank H. Vosmeer for making the angioscope available. Special thanks to J. M. P. G. Ernst, MD, PhD, for scoring the angiograms.

\section{References}

1. Berger PB, Alderman EL, Nadel A, Schaff HV. Frequency of early occlusion and stenosis in a left internal mammary artery to left anterior descending artery bypass graft after surgery through a median sternotomy on conventional bypass. Benchmark for minimally invasive direct coronary artery bypass. Circulation. 1999;100:2353-8.

2. Chaux A, Lee ME, Blanche C, Kass RM, Sherman TC, Hickey AE, et al. Intraoperative coronary angioscopy. Technique and results in the initial 58 patients. J Thorac Cardiovasc Surg. 1986;92:972-6.

3. Siegel SB, White GH, Colman PD, Nelson RJ. Intraoperative angioscopy for coronary bypass surgery. J Card Surg. 1995;10:210-20.

4. Wolf RK, Falk V. Intraoperative assessment of coronary artery bypass grafts [editorial]. J Thorac Cardiovasc Surg. 2003;126:634-7.

5. Izzat MB, Khaw KS, Atassi W, Yim AP, Wan S, El-Zufari MH. Routine intraoperative angiography improves the early patency of coronary grafts performed on the beating heart. Chest. 1999;115:98790.

6. Hiratzka LF, McPherson DD, Brandt B 3rd, Lamberth WC Jr, Sirna S, Marcus ML, et al. The role of intraoperative high-frequency epicardial echocardiography during coronary artery revascularization. Circulation. 1987;76(Suppl V):V33-8.

7. Eikelaar JH, Meijer R, van Boven WJ, Klein P, Gründeman PF, Borst C. Epicardial $10-\mathrm{MHz}$ ultrasound in off-pump coronary bypass surgery: a clinical feasibility study using a minitransducer. J Thorac Cardiovasc Surg. 2002;124:785-9.

8. Dessing TC, Budde RP, Meijer R, Bakker PF, Borst C, Gründeman PF. Geometry assessment of coronary artery anastomoses with construction errors by epicardial ultrasound. Eur J Cardiothorac Surg. 2004; 26:257-61.

9. Budde RP, Dessing TC, Meijer R, Bakker PF, Borst C, Gründeman PF. Robot-assisted $13 \mathrm{MHz}$ epicardial ultrasound for endoscopic quality assessment of coronary anastomoses. Interactive Cardiovascular and Thoracic Surgery. DOI:0.1016/j.icvts.2004.07.009, August 5, 2004. http://www.icvts.org.pdf/icvts380.pdf.

10. Landis JR, Koch GG. The measurement of observer agreement for categorical data. Biometrics. 1977;33:159-74.

11. D’Ancona G, Karamanoukian HL, Ricci M, Schmid S, Bergsland J, Salerno TA. Graft revision after transit time flow measurement in off-pump coronary artery bypass grafting. Eur J Cardiothorac Surg. 2000;17:287-93.

12. Reuthebuch O, Haussler A, Genoni M, Tavakoli R, Odavic D, Kadner A, et al. Novadaq SPY: intraoperative quality assessment in off-pump coronary artery bypass grafting. Chest. 2004;125:418-24. 